\title{
Amalgams of inverse semigroups and reversible two-counter machines.
}

\author{
Emanuele Rodaro, Pedro V. Silva \\ Centro de Matemática, Faculdade de Ciências \\ Universidade do Porto, 4169-007 Porto, Portugal \\ \{emanuele.rodaro,pvsilva\}@fc.up.pt
}

July 14, 2018

\begin{abstract}
We show that the word problem for an amalgam $\left[S_{1}, S_{2} ; U, \omega_{1}, \omega_{2}\right]$ of inverse semigroups may be undecidable even if we assume $S_{1}$ and $S_{2}$ (and therefore $U$ ) to have finite $\mathcal{R}$-classes and $\omega_{1}, \omega_{2}$ to be computable functions, interrupting a series of positive decidability results on the subject. This is achieved by encoding into an appropriate amalgam of inverse semigroups 2-counter machines with sufficient universality, and relating the nature of certain Schützenberger graphs to sequences of computations in the machine.
\end{abstract}

\section{Introduction}

If $S_{1}, S_{2}$ and $U$ are semigroups (groups) such that $U$ embeds into $S_{1}, S_{2}$ via two monomorphisms $\omega_{1}, \omega_{2}$ then $\left[S_{1}, S_{2} ; U, \omega_{1}, \omega_{2}\right]$ is called an amalgam of semigroups (groups). The amalgamated free product $S_{1} *_{U} S_{2}$ associated with this amalgam in the category of semigroups (groups) is defined by the usual universal diagram. The amalgam $\left[S_{1}, S_{2} ; U, \omega_{1}, \omega_{2}\right]$ is said to be strongly embeddable in a semigroup (group) $S$ if there exist injective homomorphisms $\phi_{i}: S_{i} \rightarrow S$ such that $\left.\phi_{1}\right|_{\omega_{1}(U)}=\left.\phi_{2}\right|_{\omega_{2}(U)}$ and $\phi_{1}\left(S_{1}\right) \cap \phi_{2}\left(S_{2}\right)=$ $\phi_{1}\left(\omega_{1}(U)\right)=\phi_{2}\left(\omega_{2}(U)\right)$. It is well known that every amalgam of groups embeds in a group (and hence in the amalgamated free product of the group 
amalgam) [7]. However there are many examples showing that in the category of semigroups not every amalgam of two semigroups is embeddable into a semigroup, see [6, 12]. On the other hand, T.E. Hall [5] showed that every amalgam of inverse semigroups (in the category of inverse semigroups) embeds in an inverse semigroup, and so in the corresponding amalgamated free product in the category of inverse semigroups. In general the class of embeddable amalgams of infinite semigroups behaves badly from the algorithmic point of view. In the paper [3], Birget, Margolis, and Meakin proved that the amalgamated free product of two finitely presented semigroups with solvable word problems and a nice common subsemigroup may have undecidable word problem. This result has been further strengthened by Sapir [12] by showing that an amalgamated free product of finite semigroups may have undecidable word problem. These results are in contrast to the situation for amalgamated free products of groups where the word problem is decidable (see [7]) assuming general nice conditions on the amalgam. The decidability result is a consequence of a normal form theorem for the free product with amalgamation of groups. A sort of normal form, but with a more geometric flavor, can be defined in the case of lower bounded or finite amalgams of inverse semigroups, see [2, 4]. In [2] Bennett showed that the word problem for lower bounded amalgam of inverse semigroups has decidable word problem, this is achieved by giving an ordered way to build the Schützenberger automata in such structure. Taking inspiration from Bennett's paper, Cherubini, Meakin and Piochi in [4], quite in contrast with Sapir's result, showed that the word problem for any amalgamated free product of finite inverse semigroups is decidable in the category of inverse semigroups. As already pointed out, this result has been achieved adapting nontrivially the work of Bennett to the finite case to obtain a sort of geometric normal form of a word $w$, called by the authors Core $(w)$. This fact, along with other lifting properties of Schützenberger automata for amalgams of finite inverse semigroups, gave rise to a series of papers [1, 10, 11] in the decidability direction. Although these clues can push toward a decidability result for amalgams of inverse semigroups with nice conditions, in this paper we give a result which goes in the opposite direction:

Theorem 1. The word problem for an amalgam $\left[S_{1}, S_{2} ; U, \omega_{1}, \omega_{2}\right]$ of inverse semigroups may be undecidable even if we assume $S_{1}$ and $S_{2}$ (and therefore $U)$ to have finite $\mathcal{R}$-classes and $\omega_{1}, \omega_{2}$ to be computable functions.

This is achieved by encoding into an appropriate amalgam of inverse 
semigroups a 2-counter machine having the right property, but still general enough to hold universality properties with respect to Turing machines. Then we show how the nature of certain Schützenberger graphs relates to sequences of computations in the machine.

\section{Inverse semigroups}

A semigroup $S$ is said to be inverse if, for each element $a \in S$, there is a unique element $a^{-1} \in S$ such that $a=a a^{-1} a$ and $a^{-1}=a^{-1} a a^{-1}$. A consequence of the definition is that idempotents commute in any inverse semigroup. Moreover, a natural partial order can be defined on $S$ by $a \leq b$ if and only if $a=e b$ for some idempotent $e$ of $S$.

Inverse semigroups may be regarded as semigroups of partial one-to-one transformations closed under inversion, so they arise very naturally in several areas of mathematics. More recently, also computer scientists have been paying attention to inverse semigroups for different reasons. First, the inverse of an element in an inverse semigroup can be seen as the "undo" of the action represented by the element. Second, algorithmic problems for inverse semigroups have received considerable attention in the literature during the past 30-35 years and in the cases where such problems are decidable, the analysis of the complexity of these algorithms is a quite natural issue.

The natural geometric framework to deal with algorithmic problems in an inverse semigroup generated by $X$ is the class of inverse $X$-graphs. Write $\widetilde{X}=X \cup X^{-1}$, where $X^{-1}=\left\{x^{-1}: x \in X\right\}$ is a set of formal inverses of $X$. We can extend $\cdot^{-1}$ to an involution on $\widetilde{X}^{*}$ through $\left(x^{-1}\right)^{-1}=x(x \in X)$ and $(u v)^{-1}=v^{-1} u^{-1}\left(u, v \in \widetilde{X}^{*}\right)$.

An inverse $X$-graph is a pair $\Gamma=(V, E)$ where $V=V(\Gamma)$ denotes the vertex set and $E=E(\Gamma) \subseteq V \times X \times V$ the edge set. We say that $\Gamma$ is:

- involutive if $(p, x, q) \in E \Rightarrow\left(q, x^{-1}, p\right) \in E$ for all $p, q \in V$ and $x \in \widetilde{X}$;

- deterministic if $(p, x, q),(p, x, r) \in E \Rightarrow q=r$ for all $p, q, r \in V$ and $x \in \widetilde{X}$

- connected if any two vertices can be connected through a path in $\Gamma$;

- inverse if it is involutive, deterministic and connected. 
An inverse $X$-automaton is a triple $\mathcal{A}=(\alpha, \Gamma, \beta)$ where $\Gamma$ is an inverse $X$-graph and $\alpha, \beta \in V(\Gamma)$ are respectively the initial and final state of the automaton.

The free inverse semigroup on a set $X$, denoted by $F I S(X)$, is the quotient of the free semigroup $\widetilde{X}^{+}$by the least congruence $\rho$ that makes the resulting quotient semigroup inverse (Vagner congruence). Its elements may be seen as finite birooted inverse $X$-trees usually known as Munn trees (see [9]).

An inverse semigroup presentation is a formal expression of the form $\operatorname{Inv}\langle X \mid R\rangle$, where $R \subseteq \widetilde{X}^{+} \times \widetilde{X}^{+}$. The presentation is finite if both $X$ and $R$ are finite. The inverse semigroup defined by this presentation is the quotient of $\widetilde{X}^{+}$by the congruence $\tau$ generated by $\rho \cup R$. We often represent the elements of $R$ as equalities of the form $w_{1}=w_{2}$.

In the paper [13], Stephen has extended the notion of Munn tree introducing the Schützenberger automaton $\mathcal{A}(X, R ; w)$ for a word $w \in \widetilde{A}^{+}$relative to the presentation $\operatorname{Inv}\langle X \mid R\rangle$ of $S$. The underlying graphs $S \Gamma(X, R ; w)$ of these automata are the strongly connected components of the Cayley graph of the presentation, which has $S$ as vertex set and edges $(s, x, s(x \tau))$ $(s \in S, x \in \tilde{X}$. More precisely, $S \Gamma(X, R ; w)$ is the restriction of this Cayley graph to the Green $\mathcal{R}$-class of $w \tau$ in $S$. It turns out that $S \Gamma(X, R ; w)$ is an inverse $X$-graph and we can now define the Schützenberger automaton

$$
\mathcal{A}(X, T ; w)=\left(\left(w w^{-1}\right) \tau, S \Gamma(X, T ; w), w \tau\right) .
$$

The importance of these automata stems from the fact that any two words $w, w^{\prime} \in \widetilde{X}^{+}$, represent the same element of $S$ if and only if $\mathcal{A}(X, R ; w)=$ $\mathcal{A}\left(X, R ; w^{\prime}\right)$, equivalent also to

$$
w \in L\left(\mathcal{A}\left(X, R ; w^{\prime}\right)\right) \quad \text { and } \quad w^{\prime} \in L(\mathcal{A}(X, R ; w)) .
$$

Hence Schützenberger automata are crucial for dealing with algorithmic problems. In [13] it is also provided an iterative procedure for approximating the Schützenberger automaton relative to a given presentation $\operatorname{Inv}\langle X \mid R\rangle$ of a word $w \in \widetilde{X}^{+}$via two operations, $R$-expansions and foldings. Let $\mathcal{A}$ be a finite inverse $X$-automaton. An $R$-expansion of $\mathcal{A}$ consists in adding a nonexisting path $p \stackrel{r^{\prime}}{\rightarrow} q$ to $\mathcal{A}$ (and the corresponding inverse edges) whenever $p \stackrel{r}{\longrightarrow} q$ is a path in $\mathcal{A}$ but no path $p \stackrel{r^{\prime}}{\longrightarrow} q$ is present $\left(r, r^{\prime}\right) \in R \cup R^{-1}$. A folding consists in identifying two edges starting at the same vertex and labeled by the same letter of the alphabet $\tilde{X}$. We say that $\mathcal{A}$ is $R$-closed if 
no $R$-expansion can be performed. The iterative sistematic application of $R$ expansions (considering all possible instances simultaneously) and complete folding to the Munn tree of a word $w \in \widetilde{X}^{+}$(obtained itself as the complete folding of the linear automaton $\rightarrow \bullet \stackrel{w}{\longrightarrow} \rightarrow$ ) produces a (possibly infinite) sequence of finite inverse $X$-automata

$$
\mathcal{A}_{1} \rightarrow \mathcal{A}_{2} \rightarrow \ldots \rightarrow \mathcal{A}_{j} \rightarrow \ldots
$$

whose colimit is $\mathcal{A}(X, R ; w)$. This colimit procedure can in fact be applied starting from any finite inverse $X$-automaton $\mathcal{A}$, producing as its colimit the $R$-closure of $\mathcal{A}$, i.e., the smallest (with respect to language) $R$-closed $X$-inverse automaton recognizing all words in $L(\mathcal{A})$ (see [14]). Any finite inverse $X$-automaton obtained from the Munn tree of $w$ by $R$-expansions and foldings is said to be a finite approximation of $\mathcal{A}(X, R ; w)$.

We close this section by fixing some notation for amalgams to be preserved through the whole paper. Let $S_{i}=\widetilde{X}_{i}^{+} / \tau_{i}$ be the inverse semigroup defined by $\operatorname{Inv}\left\langle X_{i} \mid R_{i}\right\rangle$ for $i=1,2$ with $X_{1} \cap X_{2}=\emptyset$. Let $\lambda_{i}: S_{i} \rightarrow \widetilde{X}_{i}^{+}$be a map such that, for each $s \in S_{i},\left(\lambda_{i}(s)\right) \tau_{i}=s(i=1,2)$. Finally, let $U$ be an inverse semigroup and $\omega_{i}: U \rightarrow S_{i}$ a monomorphism for $i=1,2$. The free product with amalgamation $S_{1} *_{U} S_{2}$ associated to the amalgam $\left[S_{1}, S_{2} ; U, \omega_{1}, \omega_{2}\right]$ is the inverse semigroup presented by

$$
\operatorname{Inv}\left\langle X_{1} \cup X_{2} \mid R_{1} \cup R_{2} \cup R_{3}\right\rangle,
$$

where $R_{3}=\left\{\left(\lambda_{1}\left(\omega_{1}(u)\right), \lambda_{2}\left(\omega_{2}(u)\right)\right) \mid u \in U\right\}$.

\section{Counter machines}

Definition 1. A k-counter machine (for short, $C M(k)$ ) is a system

$$
\mathcal{M}=(Q, \delta, \iota, f)
$$

where $k$ is the number of tapes, $Q$ is the nonempty finite set of internal states, $\iota \in Q$ is the initial state, and $f \in Q$ is the final (halting) state. The machine $\mathcal{M}$ uses $A=\{\perp, a\}$ as a tape alphabet ( $\perp$ is a blank symbol), $\delta$ is a move relation which is a subset of $(Q \times\{1, \ldots, k\} \times A \times Q) \cup(Q \times\{1, \ldots, k\} \times D \times Q)$ where $D=\{-, 0,+\}$ and the symbols $-, 0,+$ denote respectively left-shift, noshift, and right-shift of a head of the machine. Tapes are one-way (rightward) infinite. The leftmost squares of the tapes contain the blank symbol $\perp$, and all the other squares contain the symbol a. 
Each element of $\delta$ is thus a quadruple of one of the two forms:

$$
\left(q, i, s, q^{\prime}\right),\left(q, i, d, q^{\prime}\right)
$$

where $q, q^{\prime} \in Q, i \in\{1, \ldots, k\}, s \in A$ and $d \in D$. A quadruple of the form $\left(q, i, s, q^{\prime}\right)$ means that if $\mathcal{M}$ is in the state $q$ and the $i$ th-head is reading the symbol $s$ then the machine changes its state into $q^{\prime}$. This instruction is used to test whether the content of a counter is zero (the head is reading the symbol $\perp$ ) or positive (the head is reading a square with symbol $a$ ). We call this kind of instructions test instructions. On the other hand an instruction $\left(q, i, d, q^{\prime}\right)$ means that if $\mathcal{M}$ is in the state $q$ then:

- it shifts the $i$ th-head one cell to the right if $d=+$,

- it shifts the $i$ th-head one cell to the left if $d=-$,

- it keeps the $i$ th-head at the same cell if $d=0$,

and changes its state into $q^{\prime}$. The evolution of a $C M(k) \mathcal{M}$ can be followed through instantaneous descriptions of the machine:

Definition 2. An instantaneous description (for short, ID) of a $C M(k)$ $\mathcal{M}=(Q, \delta, \iota, f)$ is a $(k+1)$-tuple $\left(q, n_{1}, \ldots, n_{k}\right) \in Q \times \mathbb{N}^{k}$. It represents that $\mathcal{M}$ is in state $q$ and the ith-head is in position $n_{i}$ for $i=1, \ldots, k$, where we assume the position of the leftmost square of a tape to be 0 . The transition relation $\vdash_{\mathcal{M}}$ is defined as follows:

$$
\left(q, n_{l}, \ldots, n_{i-1}, n_{i}, n_{i+1} \ldots, n_{k}\right) \vdash_{\mathcal{M}}\left(q^{\prime}, n_{l}, \ldots, n_{i-1}, n_{i}^{\prime}, n_{i+1} \ldots, n_{k}\right)
$$

holds if one of the following conditions is satisfied:

1. $\left(q, i, \perp, q^{\prime}\right) \in \delta$ and $n_{i}=n_{i}^{\prime}=0$.

2. $\left(q, i, a, q^{\prime}\right) \in \delta$ and $n_{i}=n_{i}^{\prime}>0$.

3. $\left(q, i,-, q^{\prime}\right) \in \delta$ and $n_{i}-1=n_{i}^{\prime}$.

4. $\left(q, i, 0, q^{\prime}\right) \in \delta$ and $n_{i}=n_{i}^{\prime}$.

5. $\left(q, i,+, q^{\prime}\right) \in \delta$ and $n_{i}+1=n_{i}^{\prime}$.

We denote the reflexive and transitive closure of $\vdash_{\mathcal{M}}$ by $\vdash_{\mathcal{M}}^{*}$ and the $n$-step transition relation by $\vdash_{\mathcal{M}}^{n}$. 
Definition 3. Let $\mathcal{M}=(Q, \delta, \iota, f)$ be a $C M(k)$. We say that the $k$-tuple $\left(n_{1}, \ldots, n_{k}\right) \in \mathbb{N}^{k}$ is accepted by $\mathcal{M}$ if

$$
\left(\iota, n_{1}, \ldots, n_{k}\right) \vdash_{\mathcal{M}}^{*}\left(f, n_{1}^{\prime}, \ldots, n_{k}^{\prime}\right)
$$

for some $\left(n_{1}^{\prime}, \ldots, n_{k}^{\prime}\right) \in \mathbb{N}^{k}$.

Definition 4. Let $\mathcal{M}=(Q, \delta, \iota, f)$ be a $C M(k)$ and $\alpha_{i}=\left(p_{i}, j_{i}, x_{i}, p_{i}^{\prime}\right)$ with $i \in\{1,2\}$ be two distinct quadruples in $\delta$. We say that $\alpha_{1}, \alpha_{2}$ overlap in domain if

$$
p_{1}=p_{2} \wedge\left(j_{1} \neq j_{2} \vee x_{1}=x_{2} \vee x_{1} \in D \vee x_{2} \in D\right)
$$

We say that $\alpha_{1}, \alpha_{2}$ overlap in range if

$$
p_{1}^{\prime}=p_{2}^{\prime} \wedge\left(i_{1} \neq i_{2} \vee x_{1}=x_{2} \vee x_{1} \in D \vee x_{2} \in D\right)
$$

A quadruple $\alpha \in \delta$ is called deterministic (reversible respectively) if there is no other quadruple in $\delta$ which overlaps in domain (range) with $\alpha$. The machine $\mathcal{M}$ is called deterministic (reversible respectively) if every quadruple in $\delta$ is deterministic (reversible). It is clear from the definitions that every ID of a deterministic (reversible, respectively) $C M(k)$ has at most one ID that immediately follows (precedes) it in some computation. In this paper we focalize on reversible deterministic 2-counter machines because of their universality property. Indeed, the following Theorem holds:

Theorem 2. [8] For any deterministic Turing machine $\mathcal{T}$ there is a deterministic reversible $C M(2) \mathcal{M}$ that simulates $\mathcal{T}$.

Remark 1. A 2-counter machine $\mathcal{M}=(Q, \delta, \iota, f)$ can be sketched as a labeled graph $\mathscr{G}(\mathcal{M})$ with set of vertices $Q$ and labeled edges $\delta$ where we picture $\left(q_{1}, i, h, q_{2}\right) \in \delta$ as an arrow from $q_{1}$ to $q_{2}$ labelled by $i, h$ with $i \in\{1,2\}$ and $h \in\{a, \perp,+, 0,-\}$. It is clear from the definition that if $\mathcal{M}$ is deterministic then the only case where a vertex $q$ of $\mathscr{G}(\mathcal{M})$ may have two outgoing edges is when we have tests instructions (Fig. 1), i.e. $\left(q, i, a, q_{1}\right),\left(q, i, \perp, q_{2}\right)$ are two edges of $\mathscr{G}(\mathcal{M})$ with $i \in\{1,2\}$. Dually, if $\mathcal{M}$ is reversible then the only case where a vertex $q$ of $\mathscr{G}(\mathcal{M})$ may have two ingoing edges is when they are of the form $\left(q_{1}, i, a, q\right),\left(q_{2}, i, \perp, q\right)$ for some $i \in\{1,2\}$ (Fig. 11).

For our purpose we are interested in deterministic 2-counter machines with the following property. 

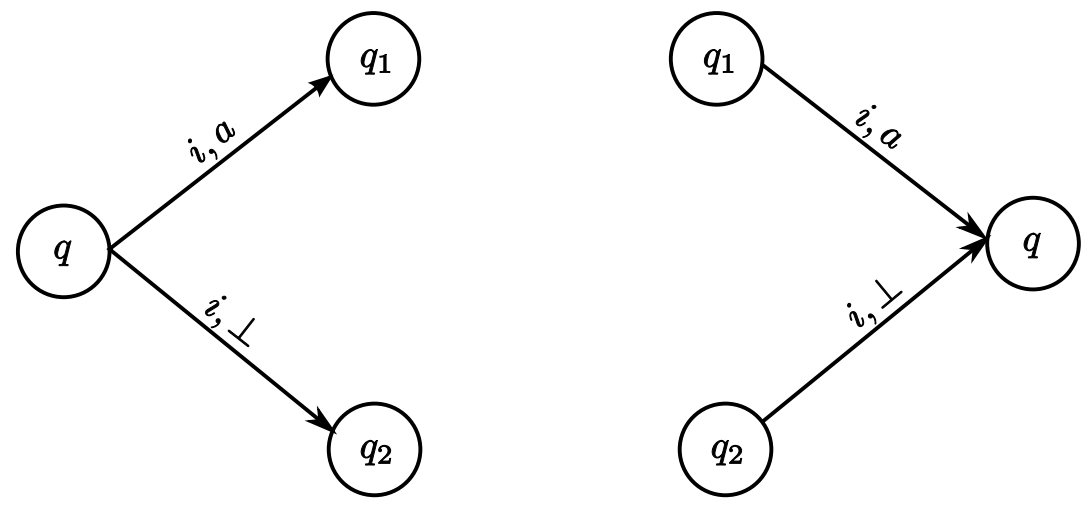

Figure 1: The deterministic case (on the left) and the reversible case (on the right).

Definition 5 (alternating 2-counter machine). Let $\mathcal{M}=(Q, \delta, \iota, f)$ be a deterministic $C M(2)$. We say that $\mathcal{M}$ is alternating if, for all pairs of different instructions $\left(q, i, h, q^{\prime}\right),\left(q^{\prime}, j, h^{\prime}, q^{\prime \prime}\right) \in \delta$, we have $j=3-i$.

We can prove the following proposition:

Proposition 1. Let $\mathcal{M}=(Q, \delta, \iota, f)$ be a deterministic reversible $C M(2)$. Then there is a deterministic reversible and alternating $C M(2) \mathcal{M}^{\prime}$ that simulates $\mathcal{M}$.

Proof. What we actually do is to add some dummy states whenever there occur two instructions $(p, i, h, q),\left(q, i, x_{1}, r_{1}\right) \in \delta$. Formally $\mathcal{M}^{\prime}=\left(Q^{\prime}, \delta^{\prime}, \iota, f\right)$ is obtained applying iteratively the following procedure. Whenever $(p, i, h, q)$, $\left(q, i, x_{1}, r_{1}\right) \in \delta$, we substitute in $\delta$ all the instructions $\left(q, i, x_{k}, r_{k}\right) \in \delta$ (at most two since $\mathcal{M}$ is deterministic) by the pairs of instructions

$$
\left(q, 3-i, 0, q_{\left(q, i, x_{k}, r_{k}\right)}\right),\left(q_{\left(q, i, x_{k}, r_{k}\right)}, i, x_{k}, r_{k}\right)
$$

where $q_{\left(q, i, x_{k}, r_{k}\right)}$ is the dummy state added. It is easy to see that the obtained $C M(2) \mathcal{M}^{\prime}$ is an alternating deterministic and reversible $C M(2)$ that simulates $\mathcal{M}$.

Note that we can always replace an instruction $(p, i, 0, q)$ by the couple of instructions $(p, i, a, q),(p, i, \perp, q)$ and on doing so the $C M(2)$ remains deterministic, reversible and alternating. Therefore, we shall assume henceforth 
that our $C M(2)$ have no instructions of type $(p, i, 0, q)$. A $C M(2)$ which is deterministic, reversible, alternating and has no instructions of the form type $(p, i, 0, q)$ is said to be normalized. Taking in particular a universal Turing machine in Theorem 2 and being undecidable whether or not a universal Turing machine can accept a given input, it follows that there exists a $C M(2)$ $\mathcal{M}_{0}$ such that it is undecidable whether or not a given $(m, n) \in \mathbb{N}^{2}$ is accepted by $\mathcal{M}_{0}$. Therefore by Proposition 1 we have the following Corollary.

Corollary 1. There exists a normalized $C M(2) \mathcal{M}^{*}$ such that it is undecidable whether or not a given $(m, n) \in \mathbb{N}^{2}$ is accepted by $\mathcal{M}^{*}$.

\section{Amalgam associated to a 2-counter machine}

In this section we associate an amalgam $\left[S_{1}, S_{2} ; U\right]$ of inverse semigroups to a deterministic reversible alternating 2-counter machine

$$
\mathcal{M}=(Q, \delta, \iota, f)
$$

The rough idea is depicted in Fig. 2, we encode the two tapes of the machine $\mathcal{M}$ by two inverse semigroups $S_{1}, S_{2}$ and the control of $\mathcal{M}$ is handled through a common inverse subsemigroup $U$.

We start by associating to $\mathcal{M}$ two inverse semigroups $S_{1}, S_{2}$, representing the two tapes, called respectively the left tape inverse semigroup and the right tape inverse semigroup of $\mathcal{M}$.

Definition 6 (left and right tape inverse semigroups). Let $\mathcal{M}=(Q, \delta, \iota, f)$ be a deterministic reversible alternating 2-counter machine. The left and right tape inverse semigroups associated to $\mathcal{M}$ are the inverse semigroups $S_{i}$ $(i=1,2)$ presented by $\operatorname{Inv}\left\langle X_{i} \mid \mathcal{T}_{i}\right\rangle$, where:

$$
\begin{gathered}
X_{i}=\left\{\perp_{i}, a_{i}, t_{i}\right\} \cup\left\{q_{i}: q \in Q\right\}, \\
\mathcal{T}_{i}=\mathcal{T}_{i}^{c} \cup \mathcal{T}_{i}^{t} \cup \mathcal{T}_{i}{ }^{w} \cup \mathcal{T}_{i}^{e} \cup \mathcal{T}_{i}^{f}
\end{gathered}
$$

and:

- $\mathcal{T}_{i}^{c}$ are the commuting relations, used to keep track of the instantaneous description of the machine;

- $\mathcal{T}_{i}^{t}$ are the test relations (corresponding to the test instructions); 


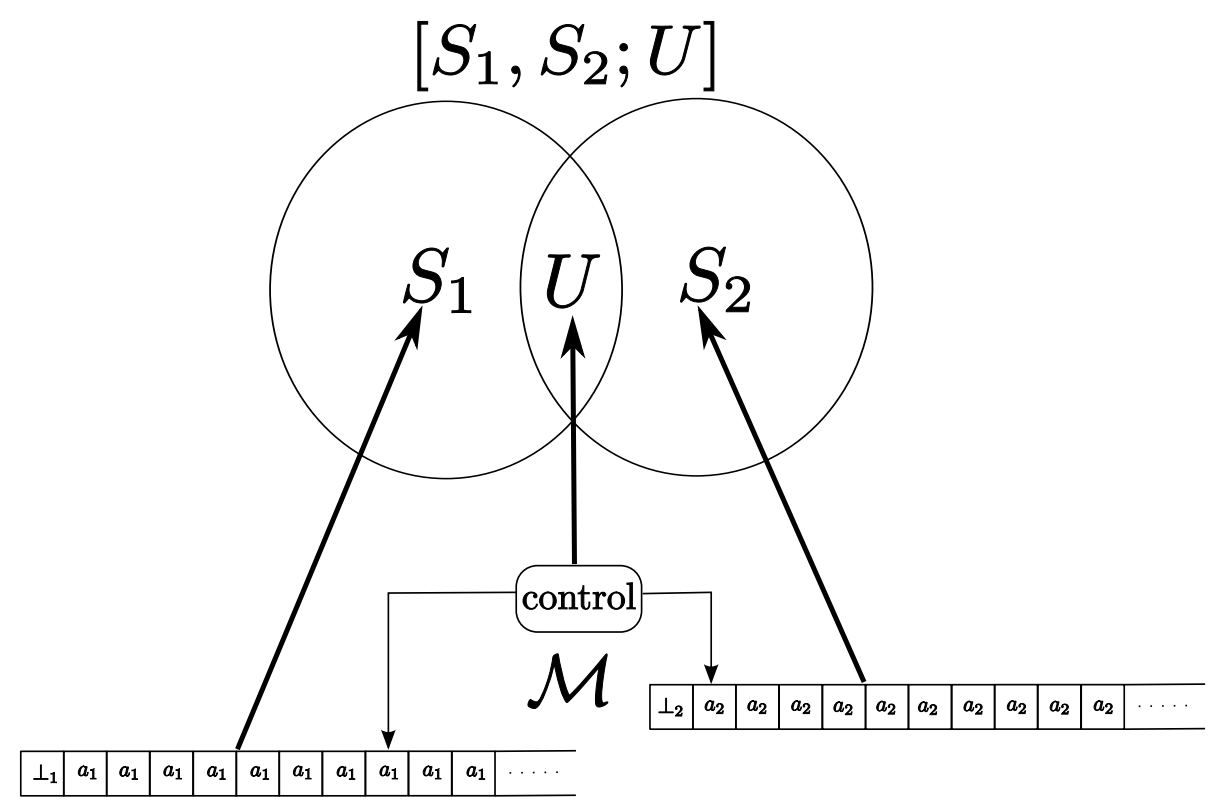

Figure 2: The rough idea of the encoding.

- $\mathcal{T}_{i}^{w}$ are the writing relations (corresponding to instructions that move the head of the ith tape to the right);

- $\mathcal{T}_{i}^{e}$ are the erasing relations (corresponding to instructions that move the head of the ith tape to the left);

- $\mathcal{T}_{i}^{f}$ are the finiteness relations (designed to enforce some finiteness properties on the semigroup $S_{i}$ ).

More precisely the relations are defined in the following way:

(i) $\mathcal{T}_{i}^{d}$ consists of all the relations of the form $t_{i} x=x t_{i}$, for $x \in\left\{a_{i}, a_{i}^{-1}, \perp_{i}\right.$ ,$\left.\perp_{i}^{-1}\right\}$ and $i=1,2$.

(ii) For each test instruction $(p, 1, s, q) \in \delta(s \in A)$, we add to $\mathcal{T}_{1}^{t}$ the relation $s p_{1}=s t_{1} q_{1} t_{1}^{-1}$.

(iii) For each test instruction $(p, 2, s, q) \in \delta(s \in A)$, we add to $\mathcal{T}_{2}^{t}$ the relation $p_{2} s=t_{2} q_{2} t_{2}^{-1} s$. 
(iv) For each right move instruction $(p, 1,+, q) \in \delta$, we add to $\mathcal{T}_{1}^{w}$ the relations $s p_{1}=s t_{1} a_{1} q_{1} t_{1}^{-1}(s \in A)$.

(v) For each right move instruction $(p, 2,+, q) \in \delta$, we add to $\mathcal{T}_{2}^{w}$ the relations $p_{2} s=t_{2} q_{2} a_{2} t_{2}^{-1} s(s \in A)$.

(vi) For each left move instruction $(p, 1,-, q) \in \delta$, we add to $\mathcal{T}_{1}^{w}$ the relations $s a_{1} p_{1}=s t_{1} q_{1} t_{1}^{-1}(s \in A)$.

(vii) For each left move instruction $(p, 2,-, q) \in \delta$, we add to $\mathcal{T}_{2}^{w}$ the relations $p_{2} a_{2} s=t_{2} q_{2} t_{2}^{-1} s(s \in A)$.

(viii) The set of relations $\mathcal{T}_{1}^{f}$ is formed by

$$
\begin{aligned}
& f_{1} x=x f_{1}=f_{1} \quad\left(x \in \widetilde{X_{1}}\right) \\
& q_{1} x=f_{1} \quad\left(q \in Q, x \in \widetilde{X_{1}} \backslash\left\{q_{1}^{-1}\right\}\right) \\
& p_{1}^{-1} q_{1}=f_{1} \quad(p, q \in Q \text { distinct }) \\
& a_{1} \perp_{1}^{-1}=\perp_{1}^{-1} a_{1}=f_{1} .
\end{aligned}
$$

(ix) The set of relations $\mathcal{T}_{2}^{f}$ is formed by

$$
\begin{aligned}
& f_{2} x=x f_{2}=f_{2} \quad\left(x \in \widetilde{X_{2}}\right) \\
& x q_{2}=f_{2} \quad\left(q \in Q, x \in \widetilde{X_{2}} \backslash\left\{q_{2}^{-1}\right\}\right) \\
& p_{2} q_{2}^{-1}=f_{2} \quad(p, q \in Q \text { distinct }) \\
& a_{2} \perp_{2}^{-1}=\perp_{2}^{-1} a_{2}=f_{2} .
\end{aligned}
$$

Remark 2. Clearly $f_{i}$ is a zero element for the inverse semigroup $S_{i}$. Note also that in cases (iv)-(vii) we have added two relations for a single move to force $a_{i}$ or $\perp_{i}$ to be present on both sides of each relation. This is done for reasons which will become clear when we shall introduce the inverse semigroup $U$. Indeed, this way the embeddability of $U$ into the tape inverse semigroups is verified since we avoid any nontrivial relations having only generators of $U$ in one side. On the other hand, each test instruction gives birth to a single relation (cases (ii)-(iii)).

We associate to $\mathcal{M}$ another inverse semigroup $U$ which represents its control unit: 
Definition 7 (core inverse semigroup). Let $\mathcal{M}=(Q, \delta, \iota, f)$ be a deterministic reversible alternating 2 -counter machine. The core inverse semigroup of $\mathcal{M}$ is the inverse semigroup $U$ presented by $\operatorname{Inv}\left\langle X_{U} \mid \mathcal{T}_{U}\right\rangle$, where $X_{U}=Q \cup\{t\}$ and the set of relations $\mathcal{T}_{U}$ is formed by

$$
\begin{aligned}
& f x=x f=f \quad\left(x \in \widetilde{X_{U}}\right), \\
& p q=f \quad(p, q \in Q), \\
& p q^{-1}=p^{-1} q=f \quad(p, q \in Q \text { distinct }) .
\end{aligned}
$$

Similarly to the previous cases, $U$ has a zero $f$. With the notation of Definitions 6 and 7 we can prove the following result:

Proposition 2. Let $\mathcal{M}$ be a deterministic reversible alternating 2-counter machine and let $S_{1}, S_{2}, U$ be respectively the left-right tape inverse semigroups and the core inverse semigroup of $\mathcal{M}$. The map $\omega_{i}$ defined by

$$
\omega_{i}(t)=t_{i}, \omega_{i}(q)=q_{i}(q \in Q)
$$

can be extended to a monomorphism $\omega_{i}: U \hookrightarrow S_{i}$ for $i=1,2$.

Proof. Let $u, v \in{\widetilde{X_{U}}}^{+}$and assume that $\omega_{i}(u)=\omega_{i}(v)$ in $S_{i}$. Suppose first that $\omega_{i}(u)$ is not the zero element of $S_{i}$. Then we are forbidden to use relations from $\mathcal{T}_{i}^{f}$ to transform $\omega_{i}(u)$ into $\omega_{i}(v)$. Since all the other relations of $\mathcal{T}_{i}$ involve $a_{i}$ or $\perp_{i}$, it follows that $\omega_{i}(v)$ must actually equal $\omega_{i}(u)$ in the free inverse semigroup over $X_{U}$, and so we definitely have $u=v$ in $U$. Hence we may assume that $\omega_{i}(u)=f_{i}$ in $S_{i}$. But the unique relations in $\mathcal{T}_{i}$ where neither $a_{i}$ nor $\perp_{i}$ occur are precisely (up to subscript $i$ ) those of $\mathcal{T}_{U}$, hence we may apply one of the latter relations to $u$ (possibly after some Vagner congruence relations) to get an occurrence of $f$. Thus $u=f$ in $U$. Similarly, also $v=f$ in $U$ and so $u=v$ holds in $U$ as required.

In view of Proposition 2 we can associate to a deterministic reversible alternating 2-counter machine an amalgam:

Definition 8. Let $\mathcal{M}=(Q, \delta, \iota, f)$ be a deterministic reversible alternating 2 -counter machine. The amalgam of inverse semigroups associated to $\mathcal{M}$ is the 5-tuple $\left[S_{1}, S_{2} ; U, \omega_{1}, \omega_{2}\right]$ where $S_{1}, S_{2}$ are the left-right tape inverse semigroups of $\mathcal{M}, U$ is the core inverse semigroup of $\mathcal{M}$ and $\omega_{i}: U \hookrightarrow S_{i}$ are the embeddings of Proposition 2. In this way the free product with amalgamation of the amalgam $\left[S_{1}, S_{2} ; U, \omega_{1}, \omega_{2}\right]$ associated to $\mathcal{M}$ can be presented by

$$
\operatorname{Inv}\left\langle X_{1} \cup X_{2} \mid \mathcal{T}_{1} \cup \mathcal{T}_{2} \cup \mathcal{T}_{3}\right\rangle
$$


where

$$
\mathcal{T}_{3}=\left\{\left(q_{1}, q_{2}\right): q \in Q\right\} \cup\left\{\left(t_{1}, t_{2}\right)\right\}
$$

The left-right tape inverse semigroups $S_{i}$ have the following important property:

Proposition 3. Let $\mathcal{M}$ be a deterministic reversible alternating 2-counter machine and let $S_{1}, S_{2}$ be respectively the left-right tape inverse semigroups of $\mathcal{M}$. Then the Green $\mathcal{R}$-classes of $S_{i}$ are finite for $i=1,2$.

Proof. First of all, let $\mathcal{M}^{\prime}$ be the machine $\mathcal{M}$ with counters 1 and 2 reversed. It is easy to check that $t_{2} \rightarrow t_{1}^{-1}, q_{2} \rightarrow q_{1}, a_{2} \rightarrow a_{1}, \perp_{2} \rightarrow \perp_{1}$ induces an isomorphism from $S_{2}$ onto the dual of $S_{1}^{\prime}$, hence it suffices to show that the Green $\mathcal{R}$-classes of $S_{1}$ are finite. This is of course equivalent to say that the Schützenberger graphs of $S_{1}$ are finite. To simplify notation, we drop all the subscripts 1 in $S_{1}, \mathcal{T}_{1}, X_{1}$ and its letters.

Let $w \in \widetilde{X}^{+}$and let $\left(\mathcal{A}_{k}\right)_{k}$ be the Stephen's sequence whose colimit is $\mathcal{A}(X, \mathcal{T} ; w)$. We can of course assume that $w$ does not represent the zero element of $S$, which is the single element of its $\mathcal{R}$-class. Let $m_{0}$ be the total number of edges labeled by letters of $Q$ in the Munn tree of $w$. We claim that there are at most $m_{0}$ expansions in the sequence $\left(\mathcal{A}_{k}\right)_{k}$ featuring edges labeled by elements of $Q$ ( $Q$-edges).

Indeed, let $Q^{\prime}$ consist of all $p \in Q$ such that there exists some instruction $(p, 1, \ldots, \ldots) \in \delta$, and let $Q^{\prime \prime}$ consist of all $q \in Q$ such that there exists some instruction $(\ldots, 1, \ldots, q) \in \delta$. Since $\mathcal{M}$ is alternating, we have $Q^{\prime} \cap Q^{\prime \prime}=\emptyset$. Moreover, since $\mathcal{M}$ is deterministic (respectively reversible), each element of $Q^{\prime}$ (respectively $Q^{\prime \prime}$ ) can feature a unique expansion: this is true for relations of type (ii), which are the unique outcome of a test instruction, and even if two test instructions $(p, 1, a, q),(p, 1, \perp, r)$ are present in $\delta$, the corresponding relations can never be used simultaneously for the same $p$-edge in view of the forbidden relations $a \perp^{-1}=f$ (we are assuming that $w \neq f$ in $S$ ). Similarly, the same $Q$-edge cannot feature two expansions involving relations of type (iv) or (vi), where application of both relations arising from a single instruction would ensure the appearance of a factor $a \perp^{-1}$. And of course, the relations of (viii) are altogether forbidden since $w \neq f$ in $S$.

Therefore there exists some $m_{1} \in \mathbb{N}$ such that all expansions and foldings involving $Q$-edges in $\left(\mathcal{A}_{k}\right)_{k}$ have taken place before we reach $\mathcal{A}_{m_{1}}$. 
Given $Y \subseteq X$, let $\sigma_{Y}: \widetilde{X}^{*} \rightarrow \mathbb{Z}$ be the homomorphism defined by

$$
\sigma_{Y}(x)=\left\{\begin{aligned}
1 & \text { if } x \in Y \\
-1 & \text { if } x \in Y^{-1} \\
0 & \text { if } x \in \widetilde{X} \backslash \widetilde{Y}
\end{aligned}\right.
$$

We claim that

$$
\text { If } u \text { labels a loop in some } \mathcal{A}_{k} \text {, then } \sigma_{t}(u)=0 \text {. }
$$

Indeed, this holds trivially for the Munn tree $\mathcal{A}_{1}$. Since $\sigma_{t}(r)=\sigma_{t}\left(r^{\prime}\right)$ for every $\left(r, r^{\prime}\right) \in \mathcal{T} \backslash \mathcal{T}^{f}$, this property is inherited through expansions in the Stephen's sequence (expansions featuring relations of $\mathcal{T}^{f}$ are forbidden since $w \neq f$ in $S$ ). Finally, the property is inherited through foldings as well: if $\mathcal{A}^{\prime}$ is obtained from $\mathcal{A}$ folding two edges of label $x$, then every loop in $\mathcal{A}^{\prime}$ can be lifted to a loop in $\mathcal{A}$ by successively inserting factors of the form $x^{-1} x$. Therefore (1) holds. We complete now the proof of our proposition by showing that the $\mathcal{T}^{c}$-closure of $\mathcal{A}_{m_{1}}$ is finite. Note that the $\mathcal{T}^{c}$-closure of $\mathcal{A}_{m_{1}}$ is also its $\mathcal{T}$-closure: we cannot use relations of $\mathcal{T}^{t} \cup \mathcal{T}^{w} \cup \mathcal{T}^{e}$ by definition of $m_{1}$, and we cannot use relations of $\mathcal{T}^{f}$ since $u \neq f$ in $S$.

Let $C_{1}, \ldots, C_{\ell}$ be the connected components of the automaton obtained by removing all $Q$-edges from $\mathcal{A}_{m_{1}}$ (the $c$-components). Clearly, performing $\mathcal{T}^{c}$-expansions cannot merge $c$-components, neither can folding edges with label in $\widetilde{A \cup t}$ (and folding of $Q$-edges will not occur after $\mathcal{A}_{m_{1}}$. Therefore we only need to prove that the $\mathcal{T}^{c}$-closure of a $c$-component $C_{j}$ is finite for $j=1, \ldots, \ell$. In view of (11), it is enough to prove that

(P) If $\mathcal{A}$ is a finite inverse $\widetilde{A \cup t}$-automaton where every $u \in \widetilde{A \cup t}^{*}$ labelling a loop satisfies $\sigma_{t}(u)=0$, then the $\mathcal{T}^{c}$-closure $\mathcal{B}$ of $\mathcal{A}$ is finite.

Let $n=|V(\mathcal{A})|$ and suppose that there is a path $i \stackrel{v}{\longrightarrow} j$ in $\mathcal{A}$ with $\left|\sigma_{t}(v)\right| \geq n$. We may assume that $v$ is shortest possible. Since $|v| \geq n$, the path $i \stackrel{v}{\longrightarrow} j$ must contain a loop, hence we may factor it as

$$
i \stackrel{v_{1}}{\longrightarrow} i_{1} \stackrel{u}{\longrightarrow} i_{1} \stackrel{v_{2}}{\longrightarrow} j
$$

with $v=v_{1} u v_{2}$ and $u \neq 1$. Now $\sigma_{t}(u)=0$ yields $\sigma_{t}\left(v_{1} v_{2}\right)=\sigma_{t}(u)$ and so $v_{1} v_{2}$ is a shorter alternative to $u$, a contradiction. Thus $\left|\sigma_{t}(v)\right|<n$ whenever $v$ labels a path in $\mathcal{A}$. We claim that also

$$
\left|\sigma_{t}(v)\right|<n \text { whenever } v \text { labels a path in } \mathcal{B} \text {. }
$$


Indeed, it suffices to remark that this property is inherited through $\mathcal{T}^{c}$ expansions and foldings, using the same argument in the proof of (11).

Assume that $i_{1}, \ldots, i_{r}$ are the vertices of $\mathcal{B}$ corresponding to the starting points of edges labelled by $a$ or $\perp$ in $\mathcal{A}$. We claim that if $i \stackrel{s}{\longrightarrow} j$ is an edge of $\mathcal{B}$ with $s \in A$, then there exists a path $i_{k} \stackrel{v}{\longrightarrow} i$ in $\mathcal{B}$ for some $k \in\{1, \ldots, r\}$ and $v \in \widetilde{t}^{*}$. Once again, it suffices to check that this property is inherited through $\mathcal{T}^{c}$-expansions and foldings, a quite trivial observation. Since $\mathcal{B}$ is inverse, we may in fact assume that $v \in t^{*} \cup\left(t^{-1}\right)^{*}$. In view of (2), it follows that there are only finitely many $A$-edges in $\mathcal{B}$, and again by (2), the number of $t$-edges must also be finite. Therefore $(\mathrm{P})$ holds and the proposition is proved.

\section{Undecidability of the word problem for amal- gams}

In this section we prove that the word problem for an amalgam $\left[S_{1}, S_{2} ; U, \omega_{1}, \omega_{2}\right]$ of inverse semigroups is undecidable even if we assume $S_{1}$ and $S_{2}$ (and therefore $U$ ) to have finite $\mathcal{R}$-classes and $\omega_{1}, \omega_{2}$ to be computable functions. The idea is to relate the computation of a deterministic reversible alternating 2-counter machine with the Schützenberger graphs of the associated amalgam.

We fix now a normalized $C M(2) \mathcal{M}=(Q, \delta, \iota, f)$ and consider the semigroup $S$ defined by the associated amalgam $\left[S_{1}, S_{2} ; U, \omega_{1}, \omega_{2}\right]$. Write $X=$ $X_{1} \cup X_{2}$ and $\mathcal{T}=\mathcal{T}_{1} \cup \mathcal{T}_{2} \cup \mathcal{T}_{3}$. Clearly $f_{1}$ and $f_{2}$ represent both the zero of $S$. Let $(m, n) \in \mathbb{N}^{2}$ and write $w_{m, n}=\perp_{1} a_{1}^{m} \iota_{1} a_{2}^{n} \perp_{2}$. Suppose that

$$
(\iota, m, n)=\left(q^{(0)}, m_{0}, n_{0}\right) \vdash_{\mathcal{M}} \ldots \vdash_{\mathcal{M}}\left(q^{(k)}, m_{k}, n_{k}\right) .
$$

Since $\mathcal{M}$ is deterministic, there is at most one such sequence of a given length starting with $(\iota, m, n)$. Write $m_{k}^{\prime}=\max \left\{m_{0}, \ldots, m_{k}\right\}, n_{k}^{\prime}=\max \left\{n_{0}, \ldots, n_{k}\right\}$. We define a finite inverse $X$-automaton $\mathcal{B}_{m, n}^{(k)}$ as follows (describing only the edges with positive label):

- The vertices are of the form $c_{i, j}$ and $d_{i, \ell}$ for $i=0, \ldots, k$ and $j=$ $0, \ldots, m_{k}^{\prime}+1$ and $\ell=0, \ldots, n_{k}^{\prime}+1$.

- $c_{0,0}$ is initial and $d_{0,0}$ is final. 


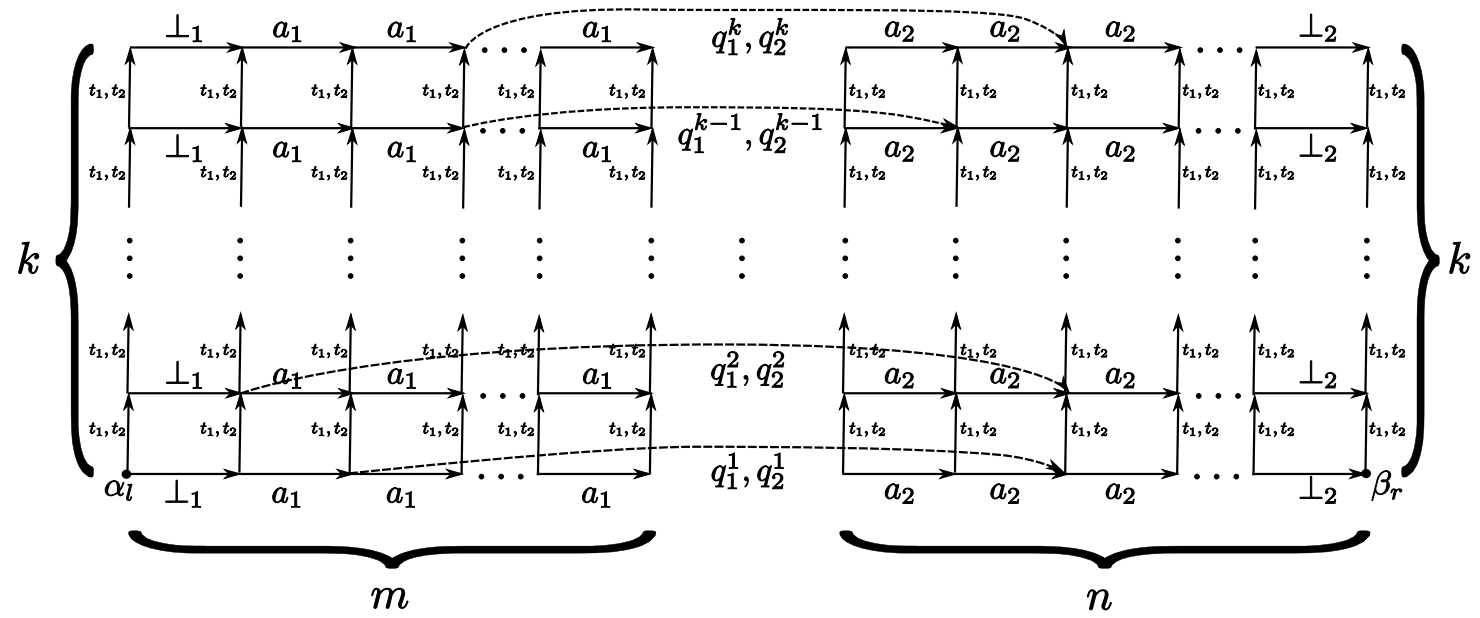

Figure 3: The automaton $\mathcal{B}_{m, n}^{(k)}$

- There exist edges $c_{i-1, j} \stackrel{t_{1}, t_{2}}{\longrightarrow} c_{i, j}$ for all $i=1, \ldots, k$ and $j=0, \ldots, m_{k}^{\prime}+1$.

- There exist edges $d_{i-1, \ell} \stackrel{t_{1}, t_{2}}{\longrightarrow} d_{i, \ell}$ for all $i=1, \ldots, k$ and $\ell=0, \ldots, n_{k}^{\prime}+1$.

- There exist edges $c_{i, 0} \stackrel{\perp_{1}}{\longrightarrow} c_{i, 1}$ for all $i=0, \ldots, k$.

- There exist edges $c_{i, j} \stackrel{a_{1}}{\longrightarrow} c_{i, j+1}$ for all $i=0, \ldots, k$ and $j=1, \ldots, m_{k}^{\prime}$.

- There exist edges $d_{i, 1} \stackrel{\perp_{2}}{\longrightarrow} d_{i, 0}$ for all $i=0, \ldots, k$.

- There exist edges $d_{i, j+1} \stackrel{a_{2}}{\longrightarrow} d_{i, j}$ for all $i=0, \ldots, k$ and $j=1, \ldots, n_{k}^{\prime}$.

- There exist edges $c_{i, m_{i}+1} \stackrel{q_{1}^{(i)}, q_{2}^{(i)}}{\longrightarrow} d_{i, n_{i}+1}$ for all $i=0, \ldots, k$.

Lemma 1. Let $\mathcal{M}$ be a normalized $C M(2)$ and let $m, n, k \in \mathbb{N}$. Then $\mathcal{B}_{m, n}^{(k)}$ is a finite approximation of $\mathcal{A}\left(X, \mathcal{T} ; w_{m, n}\right)$.

Proof. We use induction on $k$. Clearly, $\mathcal{B}_{m, n}^{(0)}$ is the Munn tree of $w_{m, n}$ with the edge $c_{0, m+1} \stackrel{\iota_{2}}{\longrightarrow} d_{0, n+1}$ adjoined. Since $\iota_{1}=\iota_{2}$ is a relation of $\mathcal{T}$, the lemma holds for $k=0$.

Assume now that $k>0$ and $\mathcal{B}_{m, n}^{(k-1)}$ is a finite approximation of the Schützenberger automaton $\mathcal{A}\left(X, \mathcal{T} ; w_{m, n}\right)$. Assume that

$$
(\iota, m, n) \vdash_{\mathcal{M}}^{k-1}\left(q^{(k-1)}, m_{k-1}, n_{k-1}\right) \vdash_{\mathcal{M}}\left(q^{(k)}, m_{k}, n_{k}\right) .
$$


Then $\left(q^{(k-1)}, i, x, q^{(k)}\right) \in \delta$ for some $i \in\{1,2\}$ (assume $i=1$ for simplicity, the case $i=2$ being analogous) and

$$
x=\left\{\begin{array}{cc}
a & \text { if } m_{k}=m_{k-1}>0 \\
\perp & \text { if } m_{k}=m_{k-1}=0 \\
+ & \text { if } m_{k}=m_{k-1}+1 \\
- & \text { if } m_{k}=m_{k-1}-1
\end{array}\right.
$$

The instruction $\left(q^{(k-1)}, 1, x, q^{(k)}\right)$ produces a relation $s y q_{1}^{(k-1)}=s t_{1} z q_{1}^{(k)} t_{1}^{-1}$ in $\mathcal{T}_{1}^{t} \cup \mathcal{T}_{1}^{w} \cup \mathcal{T}_{1}^{e}$ with $y=a_{1}$ if $x=-$ and $y=1$ otherwise, and $z=a_{1}$ if $x=+$ and $z=1$ otherwise. Using $s=\perp_{1}$ or $a_{1}$ according to the values of $m_{k-1}$ and $y$, we can now use this relation to perform an expansion of the path

$$
c_{k-1, m_{k-1}-|y|} \stackrel{s y q_{1}^{(k-1)}}{\longrightarrow} d_{k-1, n_{k-1}+1}
$$

to get after subsequent folding the edges

$$
c_{k, m_{k}+1} \stackrel{q_{1}^{(k)}}{\longrightarrow} d_{k, n_{k}+1}, c_{k-1, m_{k}+\varepsilon} \stackrel{t_{1}}{\longrightarrow} c_{k, m_{k}+\varepsilon}
$$

for $\varepsilon=0$ or $1, d_{k-1, n_{k}+1} \stackrel{t_{1}}{\longrightarrow} d_{k, n_{k}+1}$ and possibly also an edge of the form $c_{k, m_{k}} \stackrel{a_{1}}{\longrightarrow} c_{k, m_{k}+1}$. It is straightforward to check that, applying now the relations from $\mathcal{T}_{1}^{c} \cup \mathcal{T}_{2}^{c}$, followed by $t_{1}=t_{2}$ and $q_{1}^{(k)}=q_{2}^{(k)}$, we get precisely $\mathcal{B}_{m, n}^{(k)}$. Note that if $m_{k}=m_{k-1}^{\prime}+1$, we get an edge $c_{k, m_{k-1}^{\prime}+1} \stackrel{a_{1}}{\longrightarrow} c_{k, m_{k}^{\prime}+1}$ through the expansion, and the application of the relations from $\mathcal{T}_{1}^{c}$ yields indeed the required extra column of $\mathcal{B}_{m, n}^{(k)}$ with respect to $\mathcal{B}_{m, n}^{(k-1)}$. Therefore $\mathcal{B}_{m, n}^{(k)}$ is a finite approximation of $\mathcal{A}\left(X, \mathcal{T} ; w_{m, n}\right)$ and the lemma is proved.

It follows from the definition that $\mathcal{B}_{m, n}^{(k-1)}$ embeds in $\mathcal{B}_{m, n}^{(k)}$ for every $k \geq 1$. Therefore we can define $\mathcal{B}_{m, n}$ as the colimit of the sequence $\left(\mathcal{B}_{m, n}^{(k)}\right)_{k}$, where all the $\mathcal{B}_{m, n}^{(k)}$ embed. This colimit may be finite or infinite, depending on whether or not the computation in $\mathcal{M}$ halts when we start with the configuration $(\iota, m, n)$.

Let $\mathcal{C}$ denote the finite complete inverse $X$-automaton with a single vertex.

Proposition 4. Let $\mathcal{M}$ be a normalized $C M(2)$ and let $m, n \in \mathbb{N}$. Then

$$
\mathcal{A}\left(X, \mathcal{T} ; w_{m, n}\right)= \begin{cases}\mathcal{C} & \text { if }(m, n) \text { is accepted by } \mathcal{M} \\ \mathcal{B}_{m, n} & \text { otherwise }\end{cases}
$$


Proof. First of all, we claim that $\mathcal{B}_{m, n}$ is closed under all relations of $\mathcal{T} \backslash$ $\left(\mathcal{T}_{1}^{f} \cup \mathcal{T}_{2}^{f}\right)$. This is immediate for all the relations in $\mathcal{T}_{1}^{c} \cup \mathcal{T}_{2}^{c} \cup \mathcal{T}_{3}$. Since $\mathcal{M}$ is deterministic, reversible and alternating, it is also true for relations in $\mathcal{T}_{i}^{t} \cup \mathcal{T}_{i}{ }^{w} \cup \mathcal{T}_{i}{ }^{e}$ : no $Q$-edge can be involved in more than one expansion. For instance, reversibility prevents the appearance in line $i-1$ of some new $Q$-edge obtained through an expansion of a $Q$-edge in line $i$.

Now, if $(m, n)$ is accepted by $\mathcal{M}$, then $f$ labels some edge in $\mathcal{B}_{m, n}^{(k)}$ for some $k$ and so the relations of $\mathcal{T}_{1}^{f} \cup \mathcal{T}_{2}^{f}$ eventually collapse $\mathcal{B}_{m, n}^{(k)}$ into $\mathcal{C}$. Since $\mathcal{B}_{m, n}^{(k)}$ is a finite approximation of $\mathcal{A}\left(X, \mathcal{T} ; w_{m, n}\right)$, it follows that $\mathcal{A}\left(X, \mathcal{T} ; w_{m, n}\right)=\mathcal{C}$.

Finally, assume that $(m, n)$ is not accepted by $\mathcal{M}$. It is straightforward to check that in this case $\mathcal{B}_{m, n}$ must be also closed under $\mathcal{T}_{1}^{f} \cup \mathcal{T}_{2}^{f}$, hence $\mathcal{B}_{m, n}$ is $\mathcal{T}$-closed. Since $\mathcal{B}_{m, n}^{(k)}$ is a finite approximation of $\mathcal{A}\left(X, \mathcal{T} ; w_{m, n}\right)$, we have $L\left(\mathcal{B}_{m, n}^{(k)}\right) \subseteq L\left(\mathcal{A}\left(X, \mathcal{T} ; w_{m, n}\right)\right)$ for every $k$. Hence

$$
L\left(\mathcal{B}_{m, n}\right)=\bigcup_{k \geq 0} L\left(\mathcal{B}_{m, n}^{(k)}\right) \subseteq L\left(\mathcal{A}\left(X, \mathcal{T} ; w_{m, n}\right)\right)
$$

Since $\mathcal{A}\left(X, \mathcal{T} ; w_{m, n}\right)$ is the smallest $\mathcal{T}$-closed inverse $X$-automaton recognizing $w_{m, n}$, it follows that $\mathcal{A}\left(X, \mathcal{T} ; w_{m, n}\right)=\mathcal{B}_{m, n}$ as claimed.

Since $\mathcal{C}$ is the Schützenberger automaton of the zero of $S$, we immediately get:

Theorem 3. Let $\mathcal{M}$ be a normalized $C M(2)$ and let $m, n \in \mathbb{N}$. Then $w_{m, n}=$ 0 in the associated amalgam $\left[S_{1}, S_{2} ; U, \omega_{1}, \omega_{2}\right]$ if and only if $(m, n)$ is accepted by $\mathcal{M}$.

We can now prove our main result:

Theorem 4. The word problem for an amalgam $\left[S_{1}, S_{2} ; U, \omega_{1}, \omega_{2}\right]$ of inverse semigroups may be undecidable even if we assume $S_{1}$ and $S_{2}$ (and therefore $U)$ to have finite $\mathcal{R}$-classes and $\omega_{1}, \omega_{2}$ to be computable functions.

Proof. If the word problem would be decidable in these circumstances, then, in view of Proposition 3 and Theorem 3 , we could decide whether or not a normalized $C M(2)$ accepts a given $(m, n) \in \mathbb{N}^{2}$. And the latter is undecidable, even when we consider the single $C M(2) \mathcal{M}^{*}$ of Corollary 1 .

As a consequence of Theorem 3 we have also the following: 
Theorem 5. Checking finiteness for a $\mathcal{D}$-class of an amalgam $\left[S_{1}, S_{2} ; U, \omega_{1}, \omega_{2}\right]$ of inverse semigroups may be undecidable even if we assume $S_{1}$ and $S_{2}$ (and therefore $U$ ) to have finite $\mathcal{D}$-classes.

Proof. Clearly, checking the finiteness of a $\mathcal{D}$-class of a word $w$ in $S_{1} *_{U}$ $S_{2}$ is equivalent to check whether or not the corresponding Schützenberger automaton is finite. Consider again the $C M(2) \mathcal{M}^{*}$ of Corollary 1 and the associated amalgam. By Proposition 4, if $\mathcal{A}\left(X, \mathcal{T} ; w_{m, n}\right)$ would be infinite, then $(m, n)$ is not accepted by $\mathcal{M}^{*}$. On the other hand, if $\mathcal{A}\left(X, \mathcal{T} ; w_{m, n}\right)$ would be finite, we could use the Stephen's sequence to actually compute it [13] and decide whether or not $\mathcal{M}^{*}$ accepts $(m, n)$. Therefore we cannot decide whether or not $\mathcal{A}\left(X, \mathcal{T} ; w_{m, n}\right)$ is finite.

\section{Acknowledgments}

Both authors acknowledge support from C.M.U.P., financed by F.C.T. (Portugal) through the programmes POCTI and POSI, with national and E.U. structural funds. The first-named author acknowledges also the support of the FCT project SFRH/BPD/65428/2009.

\section{References}

[1] A.Cherubini, C.Nuccio, and E. Rodaro. Multilinear equations in amalgams of finite inverse semigroups. IJAC, 21(1-2):35-59, 2011.

[2] P. Bennett. Amalgamated free product of inverse semigroups. Journal of Algebra, 198:499-537, 1997.

[3] J.C. Birget, S.W. Margolis, and J.C. Meakin. On the word problem for tensor products and amalgams of monoids. Internat. J. Algebra Comput., 9(3-4):271-294, 1999.

[4] A. Cherubini, J. Meakin, and B. Piochi. Amalgams of finite inverse semigroups. Journal of Algebra, 285:706-725, 2005.

[5] T.E. Hall. Free products with amalgamation of inverse semigroups. J. Algebra, 34:375-385, 1975. 
[6] N. Kimura. On semigroups. PhD Thesis at Tulane University of Louisiana, 1957.

[7] R.C. Lyndon and P.E. Schupp. Combinatorial Group Theory. Springer, 1977.

[8] K. Morita. Universality of a reversible two-counter machine. Theoretical Computer Science, 168:303-320, 1996.

[9] W.D. Munn. Free inverse semigroups. Proc. London Math. Soc., (3) 29:385-404, 1974.

[10] E. Rodaro. Bicyclic subsemigroups in amalgams of finite inverse semigroups. IJAC, 20(1):89-113, 2010.

[11] E. Rodaro and A. Cherubini. Decidability of word problem in Yamamura's HNN extensions of finite inverse semigroups. Semigroup Forum, 77 (2):163-186, 2008.

[12] M.V. Sapir. Algorithmic problems for amalgams of finite semigroups. Journal of Algebra, 229 (2):514-531, 2000.

[13] J.B. Stephen. Presentation of inverse monoids. Journal of Pure and Applied Algebra, 198:81-112, 1990.

[14] J.B. Stephen. Amalgamated free products of inverse semigroups. Journal of Algebra, 208:339-424, 1998. 